Article

\title{
Designs of Plasmonic Metamasks for Photopatterning Molecular Orientations in Liquid Crystals
}

\author{
Yubing Guo ${ }^{1,+}$, Miao Jiang ${ }^{1,+}$, Chenhui Peng ${ }^{1}$, Kai Sun ${ }^{2}$, Oleg Yaroshchuk ${ }^{3}$, \\ Oleg D. Lavrentovich ${ }^{1}$ and Qi-Huo Wei ${ }^{1, *}$ \\ 1 Liquid Crystal Institute, Kent State University, Kent, OH 44242, USA; yguo11@kent.edu (Y.G.); \\ mjiang4@kent.edu (M.J.); cpeng2@kent.edu (C.P.); olavrent@kent.edu (O.D.L.) \\ 2 Department of Material Science and Engineering, University of Michigan, Ann Harbor, MI 48109, USA; \\ kaisun@umich.edu \\ 3 Institute of Physics, National Academy of Sciences of Ukraine, Kyiv 03028, Ukraine; \\ o.yaroshchuk@gmail.com \\ * Correspondence: qwei@kent.edu; Tel.: +1-330-672-3020 \\ + These authors contribute equally to this work. \\ Academic Editor: Vladimir Chigrinov \\ Received: 15 December 2016; Accepted: 26 December 2016; Published: 30 December 2016
}

\begin{abstract}
Aligning liquid crystal (LC) molecules into spatially non-uniform orientation patterns is central to the functionalities of many emerging LC devices. Recently, we developed a new projection photopatterning technique by using plasmonic metamasks (PMMs), and demonstrated high-resolution and high-throughput patterning of molecular orientations into arbitrary patterns. Here we present comparisons between two different types of metamask designs: one based on curvilinear nanoslits in metal films; the other based on rectangular nanoapertures in metal films. By using numerical simulations and experimental studies, we show that the PMMs based on curvilinear nanoslits exhibit advantages in their broadband and high optical transmission, while face challenges in mask designing for arbitrary molecular orientations. In contrast, the PMMs based on nanoapertures, though limited in optical transmission, present the great advantage of allowing for patterning arbitrary molecular orientation fields.
\end{abstract}

Keywords: liquid crystals; plasmonic metamasks; photoalignment; nanofabrication

\section{Introduction}

The success of the liquid crystal (LC) display technologies relies partly on the development of the low-cost rubbing technique for aligning molecules into uniform directions. Recent efforts in aligning LC molecules into spatially non-uniform orientation patterns have led to many new liquid crystal devices and applications, such as beam steering [1,2], q-plates [3-6], geometric phase wave plates [7,8], programmable origamis [9-12], controlled placement and assembly of particles [13-16], predesigned microfluidic electrokinetic flows in LCs [17,18], and command of active matter [19]. One technique is based on photoalignments through pixel-by-pixel direct laser writing $[11,20]$. By using digital micro-mirror devices to simultaneously exposing pixels with similar targeted molecular orientations, the exposure speed of the photoalignment technique can be largely increased [21,22]. Complex periodic patterns of molecular orientations can also be obtained through photoalignments by using interference of laser beams [23,24]. Another development is to use curvilinear nanogrooves generated on polyimide films by tips of an atomic force microscope [25]. Recent studies have also extended the photoalignment techniques to lyotropic LC systems $[19,26]$. These techniques are ideal for research purposes and fast device prototyping, but face challenges for large-scale manufacturing because of the serial nature of their processes or incapability to achieve arbitrary director fields in some cases. 
In order to lift these limitations, we developed a new projection photopatterning technique for arbitrary two-dimensional LC molecular orientations, which is based on plasmonic metamasks (PMMs) [27]. In contrast to traditional photolithography, where photomasks generate spatial patterns of light intensity to induce spatially varying photochemical reactions, the PMM generates spatially varying polarization directions. The produced pattern of polarization is used to induce a photoisomerization process and the spatially varying molecular orientations in the photoalignment materials. Liquid crystal molecules in contact with the photoalignment material follow these photopatterned molecular orientations because of molecular interactions. This technique enables patterning of complex molecular orientations with one single exposure (i.e., a parallel process), and exhibits advantages in terms of high-throughput, high-repeatability, sub-micron resolutions, which promise applications in large-scale device manufacturing.

Here, we present simulation and experimental studies of two different designs of the PMMs based on nanoslits and nanoapertures, respectively. Simulation results show that the PMMs with nanoslits exhibit wider bandwidth and higher transmission than the PMMs with nanoapertures. Experimental studies show that both PMMs work well in generating spatially varying polarization patterns and thus are suitable for the projection plasmonic photopatterning of complex molecular orientations. While the nanoslit-based PMMs are largely limited to generating a few special sets of molecular patterns, the nanoaperture-based PMMs allow for almost arbitrary two-dimensional LC molecular orientations.

\section{Results}

Photoalignment originates from the anisotropic molecular orientations in photoactive materials such as azo-dyes induced by linearly polarized light [28-31]. In particular, molecules with an azobenzene group tend to orient perpendicularly to the incident polarization to minimize optical absorption. The photoalignment material used in this work is brilliant yellow, which has an absorption band in the wavelength range between $400 \mathrm{~nm}$ and $550 \mathrm{~nm}$.

To maximize the efficiency of photopatterning, the PMMs should be designed to have a high transmission and polarization contrast in a wavelength range matching the absorption band of the brilliant yellow. To avoid the diffraction effect of the PMMs and maximize the spatial resolution, the periodicity of the building units in the PMMs should be smaller than the half-wavelength of light in the substrate material. Furthermore, the PMMs should have a simple design rule for practical applications.

\subsection{Design and Simulations of Nanoslit-Based PMMs}

One design of metamasks considered here is based on nanoslits. Wire grid polarizers are well known for their capabilities to generate linearly polarized light from non-polarized light in very broad wavelength ranges [32-36]. Previous studies have shown that spatially non-uniform polarization directions can be generated by using curvilinear nanoslits in Ag films [37]. To gain a basic understanding of how the transmission of the nanoslit arrays varies with the geometric parameters, we did systematic simulations for parallel slits with different periods and slit widths. As shown schematically in Figure 1a, the simulated PMMs are composed of parallel nanoslit arrays carved in Al films. The substrate material used is fused quartz for its ultraviolet (UV) transparency. The Al film thickness is fixed at $150 \mathrm{~nm}$. Being limited by focused ion beam (FIB) milling processes in mask fabrication, we set the periodicity at $300 \mathrm{~nm}$, and varied the slit width from $150 \mathrm{~nm}$ to $190 \mathrm{~nm}$. The thickness of the substrate is set infinite to avoid the effects of waveguide modes on the simulation results. 

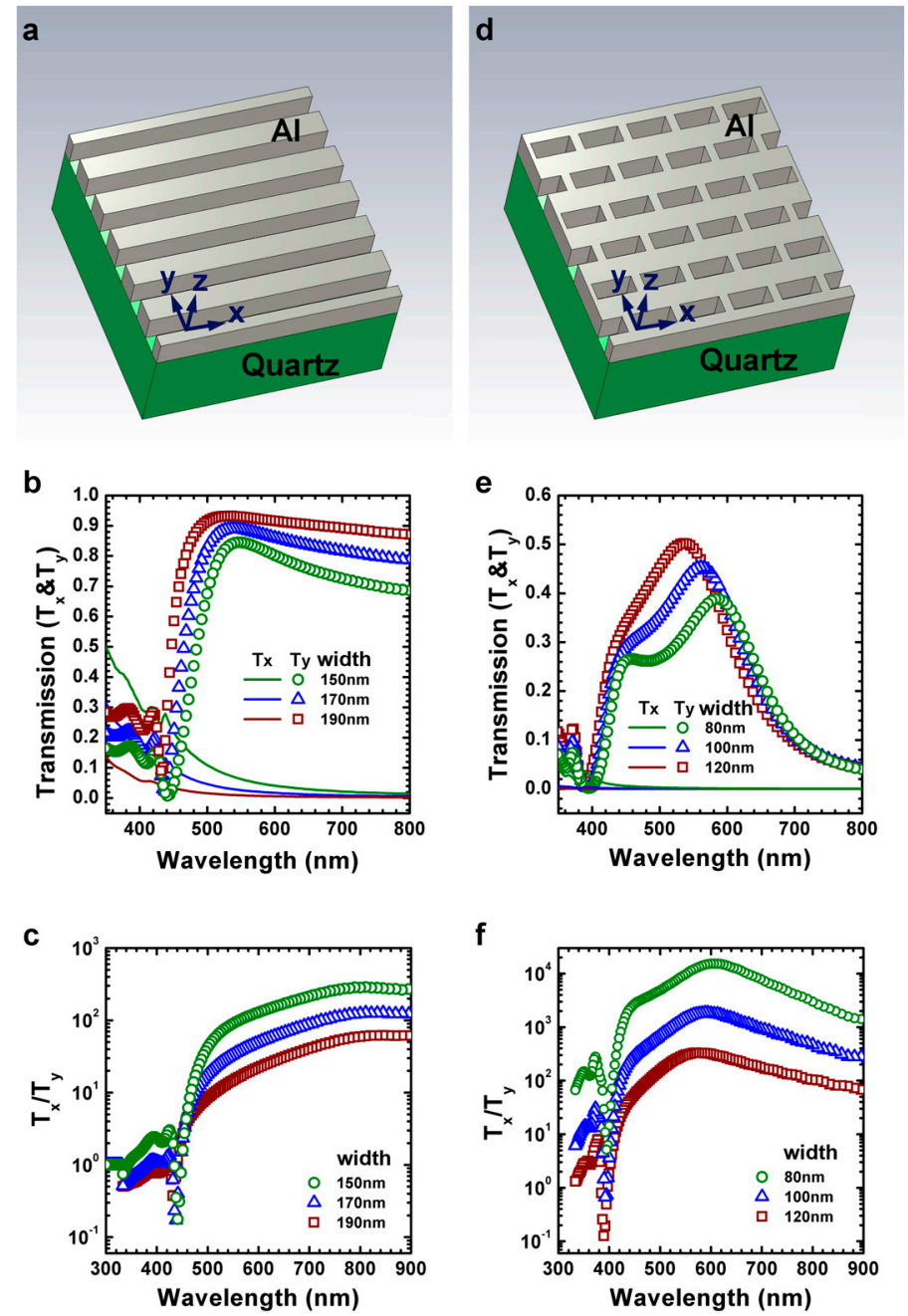

Figure 1. (a) Schematic plasmonic metamask (PMM) based on nanoslit arrays; (b,c) Simulated optical transmittance (b) and polarization ratio (c) versus wavelength for wire grid polarizer with three different slit widths: $150 \mathrm{~nm}, 170 \mathrm{~nm}, 190 \mathrm{~nm}$. The period is set at $300 \mathrm{~nm}$, and the mask film thickness is $150 \mathrm{~nm}$; (d) Schematic PMM based on rectangular nanoapertures on a triangular lattice; $(\mathbf{e}, \mathbf{f})$ Simulated spectra for optical transmittance (e) and polarization ratio (f) for nanoaperture-based PMM with three different aperture widths. The mask is of $150 \mathrm{~nm}$ in thickness and $270 \mathrm{~nm}$ in period. The nanoaperture length is $200 \mathrm{~nm}$, and width is varied: $80 \mathrm{~nm}, 100 \mathrm{~nm}, 120 \mathrm{~nm}$.

The simulation results are shown in Figure $1 b$,c. We can see that when the incident polarization is perpendicular to the slits, that is, along the $y$ direction, optical transmission $\left(\mathrm{T}_{\mathrm{y}}\right)$ can exceed $70 \%$ for wavelengths longer than $450 \mathrm{~nm}$. When the incident polarization is parallel to the slits, that is, along the $x$ direction, the optical transmission $\left(T_{\mathrm{x}}\right)$ is largely suppressed for wavelength larger than $450 \mathrm{~nm}$. It can thus be expected that the PMM illuminated with non-polarized light will transmit linearly polarized light for wavelengths longer than $450 \mathrm{~nm}$. The simulation results also show that when the slit width is increased, the optical transmission increases, the transmission band edge shifts to short wavelengths, and the polarization contrast decreases. Overall, the polarization contrast $\left(\mathrm{T}_{\mathrm{y}} / \mathrm{T}_{\mathrm{x}}\right)$ can be higher than 10 for wavelengths longer than $450 \mathrm{~nm}$, which is sufficient to induce a photoalignment effect.

The simulation results also indicate that $T_{y}$ is larger than $T_{x}$ for long wavelengths, while smaller than $T_{x}$ for short wavelengths (Figure $1 b$ ). The underlying physical mechanisms of the polarization generation have been studied and understood in a previous work on optical transmission through concentric nanoslit rings carved in Ag films [37]. A nanoslit can be considered as a 
metal-dielectric-metal (MDM) waveguide, and there exist symmetric and antisymmetric waveguide modes for both transverse magnetic (TM) and transverse electric (TE) modes. It was shown that the anti-symmetric TE mode dominates at short wavelengths, while the anti-symmetric TM mode dominates at long wavelengths [37]. In our simulations, $T_{y}$ and $T_{x}$ represent the optical transmission for the TM and TE modes, respectively. The observation that the transmitted light changes its polarization direction around the wavelength $\sim 450 \mathrm{~nm}$ are in agreements with the prior work [37].

\subsection{Design and Simulations of Nanoaperture-Based PMMs}

The second type of metamasks is based on rectangular nanoapertures, which can also create a pattern of linearly polarized light when illuminated by a non-polarized light [38-42]. As shown schematically in Figure 1d, the nanoaperture-based PMM contains rectangular nanoapertures arranged in a triangular lattice. As in the case of nanoslit-based PMMs, fused quartz is used as the substrate material, and $\mathrm{Al}$ is used as the mask material. Our simulations show that the $\mathrm{Al}$ PMMs yield higher optical transmission and allow for function at shorter wavelengths than with other plasmonic materials such as $\mathrm{Au}$ and $\mathrm{Ag}$. We calculated optical transmission and polarization contrast spectra for different aperture size and periodicity and presented these data in Reference [27]. For the completeness of the comparison between two PMM designs, we summarize our findings here.

When the mask is $150 \mathrm{~nm}$ thick and the nanoapertures are arranged in a triangular lattice with $270 \mathrm{~nm}$ periodicity, the optical transmission $\left(T_{y}\right)$ exhibits two peaks at the wavelengths between $400 \mathrm{~nm}$ and $600 \mathrm{~nm}$ for the incident polarization along the short axis of the apertures (i.e., $y$ direction), while the optical transmission $\left(T_{x}\right)$ is minimized when the polarization is along the $x$ direction. These two peaks exhibit different dependences on the nanoaperture size: the left peak is insensitive to the variation of the nanoaperture size; while the right peak shifts to the shorter wavelength when the width of the nanoaperture is increased (Figure 1e). As explained in our previous paper, these different dependences on aperture size are caused by different resonant mechanisms of these two peaks. The left peak originates in the excitation of surface plasmons at the $\mathrm{Al}-\mathrm{SiO}_{2}$ interfaces, while the right peak originates in the cavity modes inside the nanoapertures [27].

Simulations also show that reducing the periodicity leads to an increase of optical transmittance. As a trade-off between transmission efficiency and feasibility/reliability of nanofabrication processes, the PMM design was finalized with $270 \mathrm{~nm}$ periodicity, $150 \mathrm{~nm}$ thickness, and $100 \mathrm{~nm} \times 200 \mathrm{~nm}$ aperture size.

\subsection{Photopatterning with Nanoslit-Based PMMs}

Figure 2a presents an SEM image of one exemplary PMM based on nanoslits. According to the design, parallel curvilinear nanoslits with a period of $300 \mathrm{~nm}$ were carved through the $\mathrm{Al}$ film by using the focused ion beam (FIB) milling process. The final slit width turned out to be about $100 \mathrm{~nm}$, instead of the targeted $170 \mathrm{~nm}$. From the bright-field optical microscopic image (Figure 2b), the optical transmission of the nanoslit-based PMM is uniform except that the center of the concentric rings and interface between neighboring units are darker in transmission. Under a cross-polarized optical microscope, the PMM exhibits fan-textures similar to those of +1 or -1 topological defects in nematic liquid crystals (Figure 2c), which indicates that the polarization of the transmitted light is spatially non-uniform.

To characterize polarization directions of the transmitted light, we illuminate the PMM with non-polarized white light, place an analyzer between the objective and the CCD camera, and record the optical image of the PMM with the analyzer oriented at different angles $\theta$ with respect to the horizontal axis. By fitting the intensity of individual pixels with the function $I(\theta)=I_{0} \sin ^{2}\left(\theta+\theta_{0}\right)$, we can determine local polarization direction angle $\theta_{0}$ at individual pixels. The determined polarization pattern is shown in Figure 2d. It can be seen that the optical polarization direction is perpendicular to the nanoslits, in agreement with the simulations. 

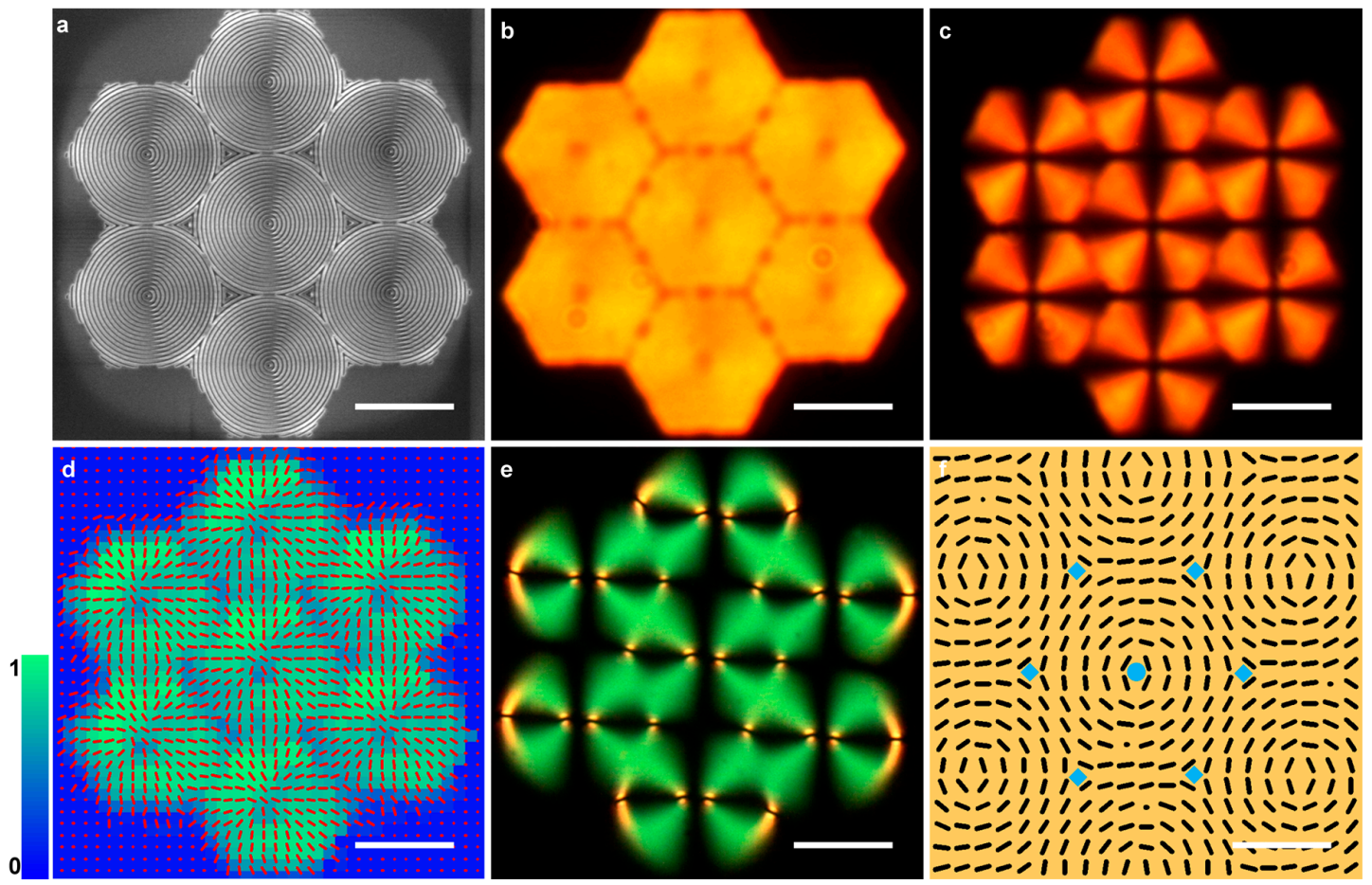

Figure 2. Exemplary PMM with curvilinear nanoslits. (a) SEM picture of a PMM; (b) Bright-field optical microscopy image of the PMM; (c) Cross-polarized optical microscopy image of the PMM; (d) Measured polarization direction for light transmitted through the PMM, the scale bar on the left hand side indicates the optical transmission; (e) Cross-polarized microscopy texture of a nematic LC in a cell patterned with the PMM; (f) Director pattern of the nematic LC cell shown in (e), the blue circle and diamonds correspond to the center of +1 and $-1 / 2$ topological defects, respectively. The scale bars are $5 \mu \mathrm{m}$ in $(\mathbf{a}-\mathbf{d})$ and $25 \mu \mathrm{m}$ in $(\mathbf{e}, \mathbf{f})$.

To test the capability of this PMM in photopatterning molecular orientation of LCs, we projected the light transmitted through the PMM onto the empty cells, thus exposing the thin films of brilliant yellow coated on the inner surfaces of the cells. After photo-exposure, the cells are filled with a nematic LC, 4-cyano-4'-pentylbiphenyl (5CB). Polarizing optical microscopy of a photo-patterned LC cell (Figure 2e) shows typical Schlieren textures with defect centers that give rise either to four or two extinction brushes. By observing the behavior of the brushes when the sample is rotated, we determine that the director pattern contains +1 and $-1 / 2$ topological defects. Further details of molecular orientations were determined by the PolScope mode of observation that allows one to map the optical retardation and in-plane orientation of the optical axis $[43,44]$. The director pattern established by PolScope observations (Figure 2f) confirms that the centers with four brushes correspond to the defect of strength +1 , and the centers with two brushes correspond to $-1 / 2$ defects. A comparison between Figures $2 \mathrm{f}$ and $2 \mathrm{a}$ indicates that the orientations of the LC molecules follow the local orientations of the nanoslit in the PMM. This yields the design rule for the nanoslit-based PMM: arranging nanoslits parallel to the desired local orientations of LC molecules.

\subsection{Photopatterning with Nanoaperture-Based PMMs}

An exemplary PMM with nanoapertures arranged in a triangular lattice is presented in Figure 3a. The long axes of the nanoapertures form arrays of +1 and $-1 / 2$ topological defects. The PMM is made of Al with $150 \mathrm{~nm}$ thickness, the nanoapertures have a $270 \mathrm{~nm}$ periodicity and $220 \mathrm{~nm} \times 120 \mathrm{~nm}$ size. Figure $3 b, c$ presents images of the PMM under a bright-field optical microscope and under a cross-polarized optical microscope, respectively. Similarly to the nanoslit-based PMM, the centers of the defects where the polarization status is undefined exhibit low optical transmission. The cross-polarized 
optical image of the PMM shows spatially non-uniform polarization-dependent optical transmission. Similarly to the process described in Section 2.3, by illuminating the PMM with non-polarized white light and recording the optical images of the PMM at different orientation angles of the analyzer, we measured the spatially varying polarization directions of the transmitted light. The results clearly show that the local polarization directions are perpendicular to the long axes of the nanoapertures, which is consistent with the simulation results (Figure $3 \mathrm{~d}$ ).

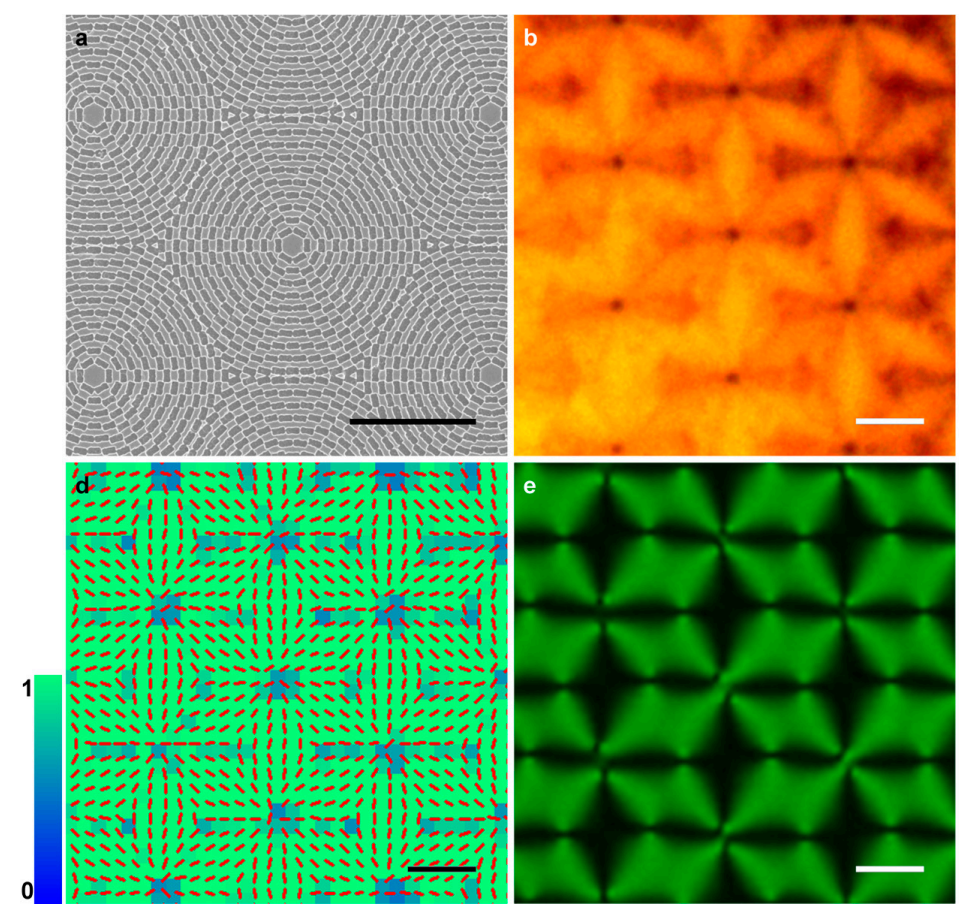

Figure 3. Exemplary PMM based on rectangular nanoapertures. (a) SEM picture of a PMM; (b) Bright-field microscopy image of the PMM; (c) Cross-polarized microscopy image of the PMM; (d) Spatial polarization state of the light transmitted through the PMM. The scale bar on the left hand side indicates the optical transmission; (e) A polarizing microscopy texture of a nematic LC cell patterned with the PMM; (f) Measured director field of the nematic LC, blue circle and diamonds correspond to the centers of +1 and $-1 / 2$ topological defects, respectively. The scale bars are $2.5 \mu \mathrm{m}$ in (a-d) and $25 \mu \mathrm{m}$ in $(\mathbf{e}, \mathbf{f})$.

The capability of this PMM in photopatterning LC molecular orientation was verified using the projection photopatterning system developed by our group. Figure 3e presents a polarizing microscopy texture of an LC cell with photoalignment layer patterned using a PMM with similar design as in Figure 3a. By rotating the sample with the Schlieren texture, we identified +1 and $-1 / 2$ topological defects. The mapped LC molecular orientation in the patterned LC cell (Figure 3f) demonstrates that the alignment directions of the photopatterned brilliant yellow layer closely follow the orientation of the nanoaperture long axes in the PMM. This implies that to design nanoaperture-based PMMs, one needs to arrange the nanoapertures with their long axes being parallel to the targeted molecular orientation, which is an extremely simple design rule. This simple rule allows one to produce practically any complex two-dimensional LC director field.

\section{Discussion}

As demonstrated by the simulations and experiments above, both nanoslit-based and nanoaperture-based PMMs can be used to generate spatially varying polarization patterns, and to produce spatially non-uniform molecular orientation of liquid crystals. Ideally, the nanoslit-based PMM designs require that the molecular director fields can be represented by streamlines with equal 
spacing, so that the nanoslits in the PMMs can have uniform spacing. Two such situations are concentric circular ring and uniform director. For other spatially non-uniform molecular orientations, the design of the PMMs with nanoslits becomes non-trivial. For example, Figure 4a presents curvilinear nanoslits forming a -1 topological defect. It can be easily seen that the nanoslit lines connecting targeted molecular directors are not parallel to each other, leading to non-uniform spacing between the nanoslits, and inevitably to introductions of many ends of the nanoslits. Since the light transmitted by the tips of the nanoslits is not linearly polarized, such PMMs may not generate the desirable polarization orientation fields. The experimental result in Figure $4 \mathrm{c}$ shows that the polarization directions generated by such a nanoslit-based metamask is not always perpendicular to the local nanoslit lines in the mask.
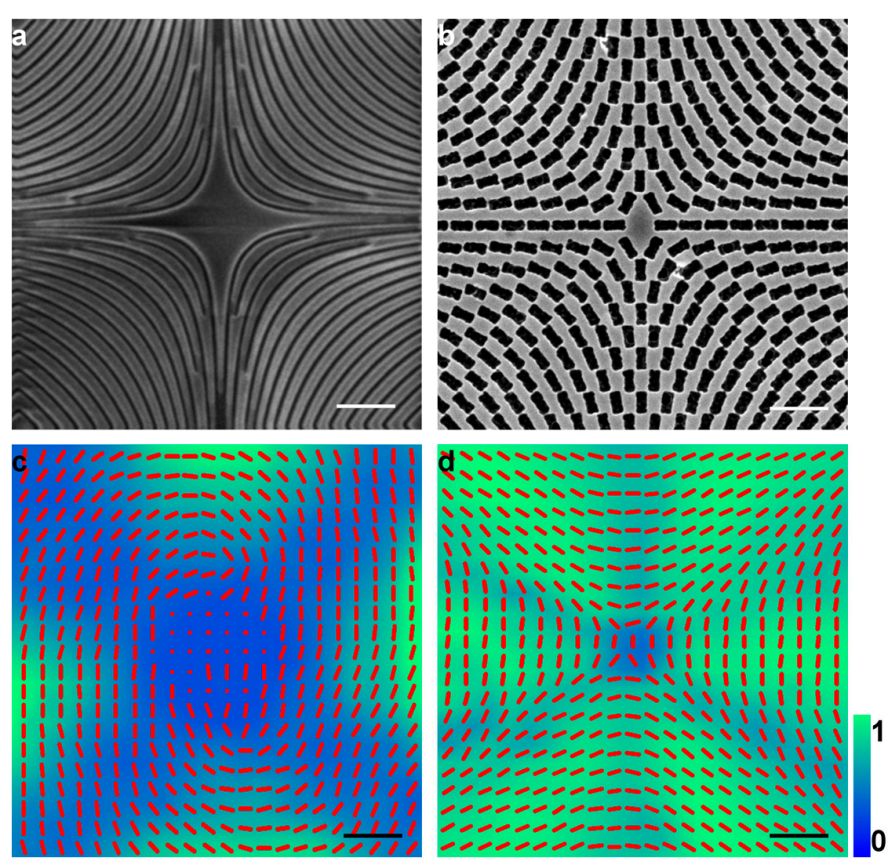

Figure 4. A comparison between the nanoslit-based and the nanoaperture-based PMMs. (a) SEM picture of a -1 defect created with the nanoslits; (b) SEM picture of a -1 defect generated by the nanoaperture arrays; (c) Measured polarization orientations and optical transmittance of the PMM in (a); (d) Measured polarization orientations and transmittance of the PMM in (b). The scale bars in (a-d) are $1 \mu \mathrm{m}$, and the scale bar on the right-hand side indicates the optical transmittance in $(\mathbf{c}, \mathbf{d})$.

The nanoaperture-based PMMs can be considered as nanoslits broken into dashed lines. Since one can adjust the orientation of a nanoaperture to match the desired molecular director without affecting its neighbors, the nanoaperture-based PMMs are more flexible in generating complex polarization and thus the molecular orientation patterns. As a comparison, we present in Figure $4 \mathrm{~b}$ a nanoaperture-based design of the PMM for the -1 topological defects. It can be seen that the nanoaperture-based PMM produces a uniform optical transmission with desired polarization directions (Figure 4d), while the nanoslit-based design does not (Figure 4c).

The other aspects to be considered in the PMM designs are the bandwidth and optical transmission. The nanoslit-based metamasks exhibit large bandwidth and the possibility to increase its transmission by using smaller periods and slit widths. In contrast, the periods and aperture sizes in the nanoaperture-based PMM designs are dictated by the transmission bands set by the absorption band of the photoalignment materials.

The spatial resolution of our plasmonic photopatterning system was shown to be below $1 \mu \mathrm{m}$ in Reference [27]. There are three factors that influence the spatial resolution of the plasmonic photopatterning technique. The first factor is related to the spatial resolution of the PMMs, that is, the periodicity of the nanoslits or the nanoapertures, which is around $300 \mathrm{~nm}$. We expected that 
the periodicity can be further improved through optimizing the mask design and nanofabrication processes. The second factor is related to the spatial resolution of our projection system. Based on the Raleigh criteria for the image systems, the spatial limit of our projection system is around $1.22 \lambda / N A$ $(\sim 1 \mu \mathrm{m})$, where $\lambda$ is light wavelength, and $N A$ is numerical aperture of objectives. With $N A=0.55$ and $\lambda=400 \mathrm{~nm}$, the resolution of the projection system is $-800 \mathrm{~nm}$. The third factor is azimuthal anchoring strength of the photoalignment materials used, which determines how well the orientation of LC molecules follow the director of the photoalignment materials. The balance between azimuthal anchoring strength $(\mathrm{W})$ and elastic constant of $\mathrm{LCs}(\mathrm{K})$ gives rise to the resolution of the photoalignment materials K/W. Shteyner and coworkers demonstrated that the photoalignment resolution of the SD1 can reach $100 \mathrm{~nm}$ [23], corresponding to an azimuthal anchoring strength in the order of $10^{-4} \mathrm{~J} / \mathrm{m}^{2}$. Therefore, the resolution limit of the plasmonic photopatterning technique is dictated by the image resolution of the projection system. By employing illuminating light with short wavelength and objectives with high numerical aperture, the resolution of plasmonic photopatterning method may be further increased.

\section{Materials and Methods}

\subsection{Fabrication of the PMMs}

To fabricate the PMMs, we explored two different fabrication processes, as shown schematically in Figure 5. In Process I (Figure 5), fused-quartz substrates are firstly cleaned with the RCA process, $15 \mathrm{~min}$ immersed in a $75^{\circ} \mathrm{C}$ solution of hydrogen peroxide, ammonium hydroxide and deionized water with 1:1:5 volume ratios. The cleaned substrates are deposited with a $5 \mathrm{~nm}$ film of Ti as an adhesion layer, then a $150 \mathrm{~nm}$ film of $\mathrm{Al}$ using an e-beam evaporation system. On top of the $\mathrm{Al}$ film, a $20 \mathrm{~nm}$-thick $\mathrm{SiO}_{2}$ is deposited by plasma-enhanced chemical vapor deposition (PECVD). The substrates are then spin-coated with $100 \mathrm{~nm}$-thick poly-methyl methacrylate (PMMA), and patterned with electron beam lithography (EBL). The patterns in PMMA are then transferred to the $\mathrm{SiO}_{2}$ layer by using reactive ion etching (RIE) with the recipe $10 \mathrm{SCCM} \mathrm{C} \mathrm{F}_{4}, 174 \mathrm{SCCM} \mathrm{He}, 4$ mTorr pressure. The patterns in the $\mathrm{SiO}_{2}$ layer are then etched through the $\mathrm{Al}$ films by using the RIE, with the recipe 30SCCM BCl $\mathrm{B}_{3}$, $15 \mathrm{SCCM} \mathrm{Cl}_{2}, 4 \mathrm{SCCM} \mathrm{CH}_{4}$, and $3 \mathrm{mTorr}$ pressure. At the end, the substrates are cleaned with acetone and water to remove the remaining PMMA.

In Process II (Figure 5), fused-quartz substrates are cleaned with RCA processes as in Process I, and deposited sequentially with $5 \mathrm{~nm}$ Ti and $150 \mathrm{~nm}$ Al film through e-beam evaporation. The designed mask patterns in the bitmap file format are then milled through the $\mathrm{Al}$ films by using a focused ion beam (FIB) system. The optimized beam voltage and current are $30 \mathrm{kV}$ and $0.23 \mathrm{nA}$, respectively.

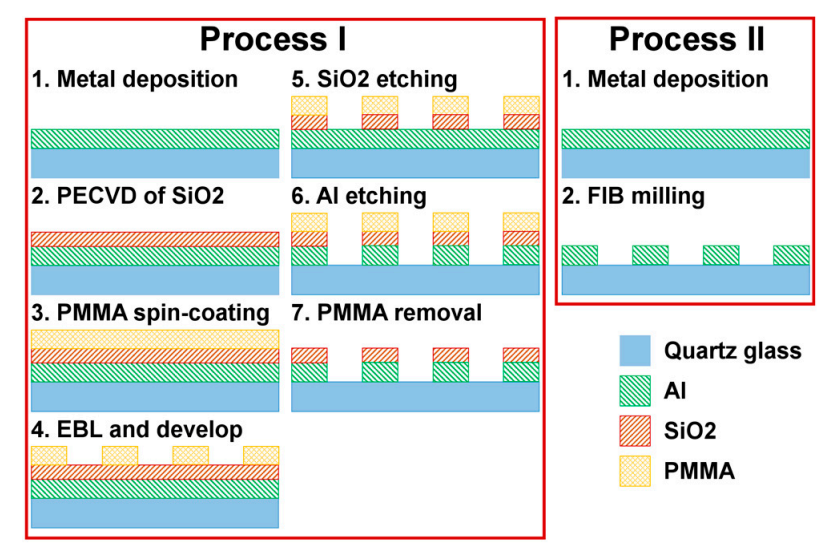

Figure 5. Fabrication process flows for the nanoaperture-based PMMs (process I) and the nanoslit-based PMMs (process II). PECVD = plasma-enhanced chemical vapor deposition; PMMA = poly-methyl methacrylate; EBL = electron beam lithography; FIB = focused ion beam. 


\subsection{Liquid Crystal Cells}

Glass substrates are cleaned in an ultrasonic bath of aqueous solution of detergents, then rinsed with deionized water and isopropanol. After blow-drying with $\mathrm{N}_{2}$ gas, the glass substrates are spin-coated with brilliant yellow (2 wt \% in N, N-Dimethylmethanamide, DMF) with the spin speed of $1500 \mathrm{rpm}$ for $30 \mathrm{~s}$, followed by hot plate baking at $95^{\circ} \mathrm{C}$ for $30 \mathrm{~min}$. Two pieces of the coated glass substrates are assembled to form a cell with the coated surface turned inward. The cell thickness is controlled by fiber spacers. For photopatterning of LC molecular orientation, light with spatially varying polarization patterns is generated by the PMMs and projected onto the middle plane of the empty cell to expose the brilliant yellow layers. When the cell thickness is smaller than the focus depth of the projection objective, the brilliant yellow at both inner surfaces is patterned at the same time. After filling the cell with 4-cyano-4'-pentylbiphenyl (5CB) through capillary suction, the 5CB molecules at the substrate surfaces follow the patterned orientations of the brilliant yellow molecules. As shown in previous studies, the brilliant yellow alignment layers yield a planar alignment of LC molecules [45].

\section{Conclusions}

In summary, we present two possible designs of plasmonic metamask for photopatterning molecular orientation of liquid crystals: one is based on curvilinear nanoslits in metal films; the other is based on rectangular nanoapertures in metal films. Our simulations and experiments have shown that both designs can generate spatially non-uniform polarization directions for aligning LC molecules into complex orientation patterns. We demonstrate that the nanoslit-based PMM designs exhibit the advantages of wide bandwidth and high transmission, but are limited to several special patterns such as uniform director and defect arrays with azimuthal director. The nanoaperture-based PMM designs have the great advantages of allowing for patterning arbitrary LC molecular orientations, though their optical transmissions are limited.

Acknowledgments: This work was supported by the National Science Foundation through CMMI-1436565 and DMR-1507637. Acknowledgment is also made to the Donors of the American Chemical Society Petroleum Research Fund for partial support of this research. Oleg Yaroshchuk was supported by the Fulbright Foundation under the program G-1-00005. Valuable discussions with Philip J. Bos, Peter Collings, Wu Lu, Carl D. Modes, Charles Rosenblatt, Timothy J. White and Hiroshi Yokoyama are acknowledged.

Author Contributions: Qi-Huo Wei conceived and designed the experiments; Yubing Guo performed the experiments; Miao Jiang performed the numerical simulations; Qi-Huo Wei, Yubing Guo and Miao Jiang analyzed the data; Kai Sun did the FIB milling and SEM; Oleg D. Lavrentovich and Chenhui Peng contributed in director pattern designing and measurements; Oleg Yaroshchuk provided guidance on photoalignment materials; Yubing Guo, Miao Jiang and Qi-Huo Wei wrote the paper with inputs from all authors.

Conflicts of Interest: The authors declare no conflict of interest.

\section{References}

1. McManamon, P.F.; Bos, P.J.; Escuti, M.J.; Heikenfeld, J.; Serati, S.; Xie, H.; Watson, E.A. A Review of Phased Array Steering for Narrow-Band Electrooptical Systems. Proc. IEEE 2009, 97, 1078-1096. [CrossRef]

2. Beeckman, J.; Neyts, K.; Vanbrabant, P.J.M. Liquid-crystal photonic applications. Opt. Eng. 2011, 50, 081202. [CrossRef]

3. Marrucci, L.; Manzo, C.; Paparo, D. Optical Spin-to-Orbital Angular Momentum Conversion in Inhomogeneous Anisotropic Media. Phys. Rev. Lett. 2006, 96, 163905. [CrossRef] [PubMed]

4. Huang, Y.-H.; Ko, S.-W.; Li, M.-S.; Chu, S.-C.; Fuh, A.Y.-G. Modulation of shape and polarization of beam using a liquid crystal q-plate that is fabricated via photo-alignment. Opt. Express 2013, 21, 10954-10961. [CrossRef] [PubMed]

5. Wei, B.Y.; Hu, W.; Ming, Y.; Xu, F.; Rubin, S.; Wang, J.G.; Chigrinov, V.; Lu, Y.Q. Generating switchable and reconfigurable optical vortices via photopatterning of liquid crystals. Adv. Mater. 2014, 26, 1590-1595. [CrossRef] [PubMed] 
6. Chen, P.; Ji, W.; Wei, B.Y.; Hu, W.; Chigrinov, V.; Lu, Y.Q. Generation of arbitrary vector beams with liquid crystal polarization converters and vector-photoaligned q-plates. Appl. Phys. Lett. 2015, 107, 241102. [CrossRef]

7. Niv, A.; Biener, G.; Kleiner, V.; Hasman, E. Propagation-invariant vectorial Bessel beams obtained by use of quantized Pancharatnam-Berry phase optical elements. Opt. Lett. 2004, 29, 238-240. [CrossRef] [PubMed]

8. Gao, K.; Cheng, H.; Bhowmik, A.K.; Bos, P.J. Thin-film Pancharatnam lens with low f-number and high quality. Opt. Express 2015, 23, 26086-26094. [CrossRef] [PubMed]

9. De Haan, L.T.; Sánchez-Somolinos, C.; Bastiaansen, C.M.W.; Schenning, A.P.H.J.; Broer, D.J. Engineering of complex order and the macroscopic deformation of liquid crystal polymer networks. Angew. Chem. Int. Ed. 2012, 51, 12469-12472. [CrossRef] [PubMed]

10. Iqbal, D.; Samiullah, M.H. Photo-responsive shape-memory and shape-changing liquid-crystal polymer networks. Materials 2013, 6, 116-142. [CrossRef]

11. Ware, T.H.; McConney, M.E.; Wie, J.J.; Tondiglia, V.P.; White, T.J. Voxelated liquid crystal elastomers. Science 2015, 347, 982-984. [CrossRef] [PubMed]

12. White, T.J.; Broer, D.J. Programmable and adaptive mechanics with liquid crystal polymer networks and elastomers. Nat. Mater. 2015, 14, 1087-1098. [CrossRef] [PubMed]

13. Muševič, I.; Škarabot, M.; Tkalec, U.; Ravnik, M.; Žumer, S. Two-Dimensional Nematic Colloidal Crystals Self-Assembled by Topological Defects. Science 2006, 313, 954-958. [CrossRef] [PubMed]

14. Peng, C.; Turiv, T.; Guo, Y.; Shiyanovskii, S.V.; Wei, Q.-H.; Lavrentovich, O.D. Control of colloidal placement by modulated molecular orientation in nematic cells. Sci. Adv. 2016, 2, e1600932. [CrossRef] [PubMed]

15. Kasyanyuk, D.; Pagliusi, P.; Mazzulla, A.; Reshetnyak, V.; Reznikov, Y.; Provenzano, C.; Giocondo, M.; Vasnetsov, M.; Yaroshchuk, O.; Cipparrone, G. Light manipulation of nanoparticles in arrays of topological defects. Sci. Rep. 2016, 6, 20742. [CrossRef] [PubMed]

16. Peng, C.; Turiv, T.; Zhang, R.; Guo, Y.; Shiyanovskii, S.V.; Wei, Q.-H.; de Pablo, J.; Lavrentovich, O.D. Controlling placement of nonspherical (boomerang) colloids in nematic cells with photopatterned director. J. Phys. Condens. Matter 2017, 29, 014005. [CrossRef] [PubMed]

17. Lavrentovich, O.D.; Lazo, I.; Pishnyak, O.P. Nonlinear electrophoresis of dielectric and metal spheres in a nematic liquid crystal. Nature 2010, 467, 947-950. [CrossRef] [PubMed]

18. Peng, C.; Guo, Y.; Conklin, C.; Viñals, J.; Shiyanovskii, S.V.; Wei, Q.-H.; Lavrentovich, O.D. Liquid crystals with patterned molecular orientation as an electrolytic active medium. Phys. Rev. E 2015, 92, 52502. [CrossRef] [PubMed]

19. Peng, C.; Turiv, T.; Guo, Y.; Wei, Q.-H.; Lavrentovich, O.D. Command of active matter by topological defects and patterns. Science 2016, 354, 882-885. [CrossRef] [PubMed]

20. McConney, M.E.; Martinez, A.; Tondiglia, V.P.; Lee, K.M.; Langley, D.; Smalyukh, I.I.; White, T.J. Topography from topology: Photoinduced surface features generated in liquid crystal polymer networks. Adv. Mater. 2013, 25, 5880-5885. [CrossRef] [PubMed]

21. Culbreath, C.; Glazar, N.; Yokoyama, H. Note: Automated maskless micro-multidomain photoalignment. Rev. Sci. Instrum. 2011, 82, 126107. [CrossRef] [PubMed]

22. Wu, H.; Hu, W.; Hu, H.; Lin, X.; Zhu, G.; Choi, J.-W.; Chigrinov, V.; Lu, Y. Arbitrary photo-patterning in liquid crystal alignments using DMD based lithography system. Opt. Express 2012, 20, 16684-16689. [CrossRef]

23. Shteyner, E.A.; Srivastava, A.K.; Chigrinov, V.G.; Kwok, H.-S.; Afanasyev, A.D. Submicron-scale liquid crystal photo-alignment. Soft Matter 2013, 9, 5160-5165. [CrossRef]

24. Du, T.; Fan, F.; Tam, A.M.W.; Sun, J.; Chigrinov, V.G.; Sing Kwok, H. Complex Nanoscale-Ordered Liquid Crystal Polymer Film for High Transmittance Holographic Polarizer. Adv. Mater. 2015, 27, 7191-7195. [CrossRef] [PubMed]

25. Murray, B.S.; Pelcovits, R.A.; Rosenblatt, C. Creating arbitrary arrays of two-dimensional topological defects. Phys. Rev. E 2014, 90, 52501. [CrossRef] [PubMed]

26. Van der Asdonk, P.; Hendrikse, H.C.; Fernandez-Castano Romera, M.; Voerman, D.; Ramakers, B.E.I.; Löwik, D.W.P.M.; Sijbesma, R.P.; Kouwer, P.H.J. Patterning of Soft Matter across Multiple Length Scales. Adv. Funct. Mater. 2016, 26, 2609-2616. [CrossRef]

27. Guo, Y.; Jiang, M.; Peng, C.; Sun, K.; Yaroshchuk, O.; Lavrentovich, O.; Wei, Q.-H. High-Resolution and High-Throughput Plasmonic Photopatterning of Complex Molecular Orientations in Liquid Crystals. Adv. Mater. 2016, 28, 2353-2358. [CrossRef] [PubMed] 
28. Ichimura, K. Photoalignment of liquid-crystal systems. Chem. Rev. 2000, 100, 1847-1873. [CrossRef] [PubMed]

29. Chigrinov, V.G.; Kozenkov, V.M.; Kwok, H.-S. Photoalignment of Liquid Crystalline Materials: Physics and Applications; John Wiley \& Sons Ltd.: Chichester, UK, 2008.

30. Kawatsuki, N. Photoalignment and Photoinduced Molecular Reorientation of Photosensitive Materials. Chem. Lett. 2011, 40, 548-554. [CrossRef]

31. Yaroshchuk, O.; Reznikov, Y. Photoalignment of liquid crystals: Basics and current trends. J. Mater. Chem. 2012, 22, 286-300. [CrossRef]

32. Yu, X.J.; Kwok, H.S. Optical wire-grid polarizers at oblique angles of incidence. J. Appl. Phys. 2003, 93, 4407-4412. [CrossRef]

33. Liu, X.; Deng, X.; Sciortino, P.; Buonanno, M.; Walters, F.; Varghese, R.; Bacon, J.; Chen, L.; O’Brien, N.; Wang, J.J. Large area, $38 \mathrm{~nm}$ half-pitch grating fabrication by using atomic spacer lithography from aluminum wire grids. Nano Lett. 2006, 6, 2723-2727. [CrossRef] [PubMed]

34. Wang, J.J.; Chen, L.; Liu, X.; Sciortino, P.; Liu, F.; Walters, F.; Deng, X. 30-nm-wide aluminum nanowire grid for ultrahigh contrast and transmittance polarizers made by UV-nanoimprint lithography. Appl. Phys. Lett. 2006, 89, 141105. [CrossRef]

35. Chen, L.; Wang, J.J.; Walters, F.; Deng, X.; Buonanno, M.; Tai, S.; Liu, X. Large flexible nanowire grid visible polarizer made by nanoimprint lithography. Appl. Phys. Lett. 2007, 90, 063111. [CrossRef]

36. Wang, J.J.; Walters, F.; Liu, X.; Sciortino, P.; Deng, X. High-performance, large area, deep ultraviolet to infrared polarizers based on $40 \mathrm{~nm}$ line/78 nm space nanowire grids. Appl. Phys. Lett. 2007, 90, 061104. [CrossRef]

37. Wang, F.; Xiao, M.; Sun, K.; Wei, Q.-H. Generation of radially and azimuthally polarized light by optical transmission through concentric circular nanoslits in Ag films. Opt. Express 2010, 18, 63-71. [CrossRef] [PubMed]

38. Garcia-Vidal, F.J.; Moreno, E.; Porto, J.A.; Martin-Moreno, L. On the transmission of light through a single rectangular hole. Phys. Rev. Lett. 2005, 95, 103901. [CrossRef] [PubMed]

39. Van der Molen, K.L.; Klein Koerkamp, K.J.; Enoch, S.; Segerink, F.B.; van Hulst, N.F.; Kuipers, L. Role of shape and localized resonances in extraordinary transmission through periodic arrays of subwavelength holes: Experiment and theory. Phys. Rev. B 2005, 72, 45421. [CrossRef]

40. Kong, F.; Wu, B.-I.; Chen, H.; Kong, J.A. Surface plasmon mode analysis of nanoscale metallic rectangular waveguide. Opt. Express 2007, 15, 12331-12337. [CrossRef] [PubMed]

41. Garcia-Vidal, F.J.; Martin-Moreno, L.; Ebbesen, T.W.; Kuipers, L. Light passing through subwavelength apertures. Rev. Mod. Phys. 2010, 82, 729-787. [CrossRef]

42. Mary, A.; Rodrigo, S.; Martín-Moreno, L.; García-Vidal, F. Holey metal films: From extraordinary transmission to negative-index behavior. Phys. Rev. B 2009, 80, 165431. [CrossRef]

43. Shribak, M.; Oldenbourg, R. Techniques for fast and sensitive measurements of two-dimensional birefringence distributions. Appl. Opt. 2003, 42, 3009-3017. [CrossRef] [PubMed]

44. Lavrentovich, O.D. Looking at the world through liquid crystal glasses. Contemp. Math. 2011, 577, $25-46$.

45. Yaroshchuk, O.; Gurumurthy, H.; Chigrinov, V.G.; Kwok, H.S.; Hasebe, H.; Takatsu, H. Photoalignment Properties of Brilliant Yellow Dye. In Proceedings of the 14th International Display Workshop, Sapporo, Japan, 5-7 December 2007; pp. 1665-1668.

(C) 2016 by the authors; licensee MDPI, Basel, Switzerland. This article is an open access article distributed under the terms and conditions of the Creative Commons Attribution (CC-BY) license (http://creativecommons.org/licenses/by/4.0/). 